\title{
On the behaviour of solutions of the two-cell cubic autocatalator reaction model
}

\author{
H. I. Abdel-Gawad* K. M. Saad \\ (Received 31 October 2001; revised 10 June 2002)
}

\begin{abstract}
Approximate solutions of the initial value problem for reaction diffusion equations in two regions (cells) are obtained. The system is considered here with two chemical species, species $A$ and the autocatalyst $B$. The reaction is taken to be cubic in the autocatalysis in the first region with linear exchange through $A$. In the first region, the autocatalyst is taken to decay linearly. Approximate solutions are found through the Picard iterative sequence of solutions. The space and time variations of the concentration of the species $A$ and $B$ are evaluated in the two regions.
\end{abstract}

*Department of Mathematics, Faculty of Science, Cairo University, Egypt. mailto:hamdyig@yahoo.com

$\dagger$ Department of Mathematics, Faculty of Science, Taiz University, Yemen. mailto:Khaledma_sd@hotmail.com

${ }^{0}$ See http: //anziamj . austms . org. au/V44/E032 for this article, (C) Austral. Mathematical Soc. 2002. Published July 5, 2002. ISSN 1446-8735 
The oscillation of the concentrations in times has been observed in different locations. This phenomena is stepped out for relatively large times. Comparison between two consecutive solutions is made. The maximum error estimate is of order $10^{-3}$ for some appropriate time period. At this time level, the solutions obtained are adequate for laboratory simulation experiments to open systems. It is observed that no initiation to travelling waves occurs whenever the initial values of the concentrations of the reactant (or the autocatalysts) are not periodic.

\section{Contents}

1 Introduction and mathematical formulation

2 Outline of the method for initial value problems

E6

3 The first approximation to the solution of the problem for $\gamma<1$

E9

4 The second approximation and maximum error estimate

E21

5 On the existence of realistic permanent travelling wave solutions

E27

6 Conclusions

E29

References 


\section{Introduction and mathematical formulation}

Chemical and biological reactions in open systems (which allow mass transport across the reaction boundary) may give rise to complex phenomena. These phenomena may be multiple stationary-states, damped oscillations (see [5]), target patterns, spiral waves [10, 11, 22] or strange attractors [8, 9]. These nonlinear phenomena may be due to feedback through the detailed chemical mechanism or through departure from the isothermal state [20]. Our aim here is to study the time and space variation of the concentrations in isothermal-reaction diffusion processes in two regions. Coupling between the two regions is assumed by allowing linear diffusive interchange by autocatalyst (or the reactant). This is achieved practically through a semi-permeable membrane interface between the two regions (cells). For more details see the experimental works in $[6,7,19]$. We consider an initial value problem where the autocatalyst is injected locally into uniform concentrations of the reactant. The initiation and propagation of travelling waves for these reactions with different orders and different geometries have been studied in a series of papers $[15,12,13,14,17,16,18]$. The travelling wave solutions describe the propagation of waves with speed $c$. Steady state solutions correspond to taking $c=0$. In general these solutions are independent of the initial conditions and they may be valid asymptotically. They may not be realistic solutions. Thus short-range phenomena are masked by these solutions. A typical phenomena consists of the oscillation of the concentrations of the reactant and the autocatalyst in the two regions. We now consider the following model. In Region I, the reaction is given by a cubic autocatalyst together with a linear decay of the autocatalyst, to an 
inert product $C$ :

$$
A+2 B \rightarrow 3 B \quad\left(\text { rate }=k_{1} A B^{2}\right), \quad \text { and } \quad B \rightarrow C \quad\left(\text { rate }=k_{2} B\right) .
$$

In Region II, the reaction is given by a pure interchange of the reactant through the two regions while the autocatalyst concentration is taken to be zero. Also, it is assumed that $A$ interchanges from one region to another through mass exchange rate $k_{3}$. The motivation for studying these problems is to investigate the series of interesting phenomena produced in the propagation of reaction diffusion waves on coupled membrane interfaces. Here we shall confine ourselves to the one-dimensional model equations which describe such autocatalytic reactions. They are (see also [17]) for $(X, \tau) \in(-\infty, \infty) \times[0, T]:$

$$
\begin{aligned}
a_{1 \tau} & =D a_{1 X X}-k_{1} a_{1} b^{2}+k_{3}\left(a_{2}-a_{1}\right), \\
b_{\tau} & =D b_{X X}+k_{1} a_{1} b^{2}-k_{2} b \\
a_{2 \tau} & =D a_{2 X X}+k_{3}\left(a_{1}-a_{2}\right) .
\end{aligned}
$$

In (1-3) $a_{1}, a_{2}$ and $b$ are the concentrations of the reactant $A$ and the autocatalyst $B$ in the two Regions I and II respectively. The diffusion coefficients of the species $A$ and $B$ in the two regions are assumed to be the same, namely $D$. The initial conditions are that $A$ is a uniform distribution in the two regions while the autocatalyst is injected locally:

$$
a_{i}(X, 0)=a_{0} ; \quad b(X, 0)=a_{0}\left\{\begin{array}{lc}
H(X), & -\ell<X<\ell \\
0, & |X| \geq \ell
\end{array}\right.
$$

where $H(X)$ is a nonnegative function which is infinitely differentiable and vanishes as $|X| \rightarrow \ell$. The boundary conditions are

$$
a_{i} \rightarrow a_{0}, \quad b \rightarrow 0 \quad \text { as } \quad|X| \rightarrow \infty, \quad t \geq 0 .
$$


By the nondimensionalisation

$$
a_{i}=a_{0} \alpha_{i}, \quad b=a_{0} \beta, \quad t=k_{1} a_{0}^{2} \tau \quad \text { and } \quad x=\left(\frac{k_{1} a_{0}^{2}}{D}\right)^{1 / 2} X,
$$

the PDEs $(1-3)$ become, for $(x, t) \in(-\infty, \infty) \times[0, T]$,

$$
\begin{aligned}
\alpha_{1 t} & =\alpha_{1 x x}-\alpha_{1} \beta^{2}+\gamma\left(\alpha_{2}-\alpha_{1}\right), \\
\beta_{t} & =\beta_{x x}+\alpha_{1} \beta^{2}-k \beta \\
\alpha_{2 t} & =\alpha_{2 x x}+\gamma\left(\alpha_{1}-\alpha_{2}\right) .
\end{aligned}
$$

In the new variables, the initial and the boundary conditions become

$$
\begin{aligned}
& \alpha_{i}(x, 0)=1, \quad \beta(x, 0)= \begin{cases}h g(x), & |x|<\ell_{1} \\
0, & |x| \geq \ell_{1},\end{cases} \\
& \alpha_{i} \rightarrow 1, \quad \beta \rightarrow 0 \quad \text { as }|x| \rightarrow \infty, \quad t \geq 0, \quad i=1,2
\end{aligned}
$$

where $\ell_{1}=\left(k_{1} a_{0}^{2} \ell^{2} / D\right)^{1 / 2}$. The non-dimensional constants $k=$ $k_{2} /\left(k_{1} a_{0}^{2}\right)$ and $\gamma=k_{3} /\left(k_{1} a_{0}^{2}\right)$ represent the strength of the autocatalyst decay and the coupling between the two regions respectively. Here the function $g(x)$ is taken as

$$
g(x)=e^{1 /\left(x^{2}-\ell_{1}^{2}\right)} .
$$

Notice that the function $\beta(x, 0)$ has compact support (or it belongs to the space $\left.C_{0}^{\infty}\left(\left[-\ell_{1}, \ell_{1}\right]\right)\right)$. Hereafter, we confine ourselves to the case $\ell_{1}=1$. By applying the scalar maximum principle to the parabolic operator, it has been shown that $0 \leq \alpha_{i} \leq 1$ and $0 \leq \beta \leq 2+h, i=1,2$. These results have been verified through numerical calculations [17]. 


\section{Outline of the method for initial value problems}

In this work, we use the approach developed in $[1,2,3]$ to find approximate solutions to the equations (6-8) for the initial value problems (IVP). The approach is based on finding the formal exact solution for the IVP. After the exact solution is found, the Picard iterative sequence of solutions is constructed. We truncate the Picard iteration at the first and second approximations for the problem under consideration. We write the set of equations (6-8) as

$$
\begin{aligned}
\mathbf{U}_{t} & =\hat{M} \mathbf{U}+\mathbf{S}(\mathbf{U}) \\
\hat{M} & =\left(\begin{array}{ccc}
\partial_{x}^{2}-\gamma & 0 & \gamma \\
0 & \partial_{x}^{2}-k & 0 \\
\gamma & 0 & \partial_{x}^{2}-\gamma
\end{array}\right), \\
\mathbf{S}(\mathbf{U}) & =\left(\begin{array}{ccc}
-\alpha_{1} \beta^{2} & \alpha_{1} \beta^{2} & 0
\end{array}\right)^{T}, \quad \mathbf{U}=\left(\begin{array}{lll}
\alpha_{1} & \beta & \alpha_{2}
\end{array}\right)^{T} .
\end{aligned}
$$

In (11) we consider the vector $\mathbf{S}$ as a source term. By using the results found in [3], the formal exact solution of the set (11-13) for the IVP is

$$
\mathbf{U}(x, t)=\mathbf{U}_{L}(x, t)+\int_{0}^{t} e^{\left(t-t_{1}\right) \hat{M}} \mathbf{S}\left(\mathbf{U}\left(x, t_{1}\right)\right) d t_{1},
$$

where $\mathbf{U}_{L}(x, t)$ is the solution of the linear problem which satisfies

$$
\mathbf{U}_{L t}(x, t)=\hat{M} \mathbf{U}_{L}(x, t) .
$$

The solution of $(15)$ is

$$
\mathbf{U}_{L}(x, t)=e^{t \hat{M}} \mathbf{U}_{0}(x),
$$

where the vector of initial conditions $\mathbf{U}_{0}(x)=(1, h g(x), 1)^{T}$. We can easily show that the solution (14-16) satisfies the system (1113) and (9). 
The exponential of a matrix of operators in $(14,16)$ is defined by

$$
e^{-\lambda \hat{M}_{i j}}=\frac{1}{2 \pi i} \int_{c-i \infty}^{c+i \infty} e^{-\lambda Z}\left(Z \hat{I}-\hat{M}_{i j}\right)^{-1} d Z,
$$

where $\left(Z \hat{I}-\hat{M}_{i j}\right)^{-1}$ is the resolvent matrix of operators. In fact the matrix of operators $\left(Z \hat{I}-\hat{M}_{i j}\right)$ may be written as $\left(Z \hat{I}-\hat{M}_{i j}\right)=(\hat{Z} I-$ $\left.\hat{M}_{i j}\right)$, where $\hat{Z}$ is an eigenoperator of the matrix of operators $\hat{M}$. The last identity holds because if $Z$ is an eigenvalue of the operator $\hat{Z}$, namely $\hat{Z} f=Z f$ and $Z \hat{I} f=Z f$ for all $f \in C^{\infty}$, then we can write $Z \hat{I} f=\hat{Z} I f$. To define an eigenoperator of a matrix of operators, consider the problem

$$
\hat{M} \mathbf{f}=M \mathbf{f} \quad \text { for all } \mathbf{f} \in C^{\infty},
$$

where $\mathbf{f}=\left(f_{1}, f_{2}, \ldots, f_{m}\right)^{T}$ and $M=\left(M_{i j}\right), i, j=1,2, \ldots, m$ is the matrix which results from operating by $\hat{M}$ onto $\mathbf{f}$. This holds by bearing in mind that the operators $M_{i j}$ have constant coefficients. Also, we consider the eigenvalue problem,

$$
\hat{L}_{i} \mathbf{f}=\lambda_{i} \mathbf{f}, \quad i=1,2, \ldots, m \quad \text { for all } \mathbf{f} \in C^{\infty} .
$$

Definition 1 The operators $\hat{L}_{i}$ are eigenoperators of the matrix of operators $\hat{M}$ if $\lambda_{i}$ are the eigenvalues of the matrix $M$.

In this case, we have

$$
\left(\hat{M}-\hat{L}_{i} I\right) \mathbf{f}=\left(M-\lambda_{i} I\right) \mathbf{f}=0 \quad \text { or } \quad\left|M-\lambda_{i} I\right|=\left|\mathbf{f}^{T}\left(\hat{M}-\hat{L}_{i} I\right) \mathbf{f}\right|=0,
$$

where $I$ is the identity matrix. By using this definition, the eigenoperators of the matrix $\hat{M}$ are $\partial_{x}^{2}, \partial_{x}^{2}-k$, and $\partial_{x}^{2}-2 \gamma$. We now 
construct the Picard iterative sequence of solutions for $n=1,2, \ldots$ of

$$
\begin{aligned}
& \mathbf{U}_{n}(x, t)=\mathbf{U}_{0}(x, t)+\int_{0}^{t} e^{\left(t-t_{1}\right) \hat{M}} \mathbf{S}_{n-1}\left(\mathbf{U}\left(x, t_{1}\right)\right) d t_{1}, \\
& \text { where } \mathbf{U}_{0}(x, t)=\mathbf{U}_{L}(x, t) \text {, } \\
& \text { and } \quad \mathbf{S}_{n-1}\left(\mathbf{U}\left(x, t_{1}\right)\right)=\mathbf{S}\left(\mathbf{U}_{n-1}\left(x, t_{1}\right)\right) \text {. }
\end{aligned}
$$

Under the initial conditions (9), we can show that the sequence $\mathbf{U}_{n}$ converges uniformly to the exact solution $\mathbf{U}$. Also, it can be shown that this solution is unique and stable. We mention the following lemmas and theorems without proof.

Lemma 2 The matrix of operators $e^{t \hat{M}}, t>0$, acts on the space $L_{1} \cap C^{\infty}$ which is closed and bounded.

Lemma 3 The matrix of operators $e^{t \hat{M}}, t>0$, acts on the space $C_{0}^{\infty}$ which is in $S_{\infty}$.

Note: as the initial function $\beta_{0}(\beta(x, 0))$ is in $C_{0}^{\infty}$, it can be shown that the solution of the linear problem, namely $\beta_{L}$, is in the space $S_{\infty}$ (which includes $C_{0}^{\infty}$ ), the space of rapidly decreasing functions which are infinitely differentiable. Consequently $\mathbf{S}_{0} \equiv \mathbf{S}\left(\mathbf{U}_{L}\right)$ is in $S_{\infty}$

Theorem 4 If $\mathbf{S}_{0} \in S_{\infty}$, is bounded and Lipschitz continuous, then the sequence $\mathbf{U}_{n}$ in (21) converges uniformly to the exact solution $\mathbf{U}$ for the initial value problem (11-13) and (9).

Theorem 5 Under these conditions, the solution (11-13) is unique and stable. 
The proofs of these theorems and lemmas follow those in [3]. For convenience, hereafter, we shall rescale time according to whether $\gamma<1$ or $\gamma \geq 1$ : we use the transformation $t \rightarrow \gamma t$ for $\gamma<1$; and $t \rightarrow t / \gamma$ for $\gamma>1$. These two cases are considered respectively.

\section{The first approximation to the solution of the problem for $\gamma<1$}

Consider the case when $\gamma<1$ and make the rescaling transformation $t \rightarrow \gamma t$. The set of equations (11-13) becomes

$$
\begin{aligned}
\mathbf{U}_{t} & =\hat{M}_{1} \mathbf{U}+\mathbf{S}_{1}(\mathbf{U}), \\
\mathbf{M}_{1} & =\gamma \mathbf{M}, \quad \mathbf{S}_{1}(\mathbf{U})=\gamma \mathbf{S}(\mathbf{U}),
\end{aligned}
$$

where $\hat{M}$ and $\mathbf{S}(\mathbf{U})$ are given by (13). The first approximation for the solution (22-23) and (9-10) is

$$
\mathbf{U}_{1}(x, t)=\mathbf{U}_{L}(x, t)+\int_{0}^{t} e^{\left(t-t_{1}\right) \hat{M}_{1}} \mathbf{S}_{1}\left(\mathbf{U}_{L}\left(x, t_{1}\right)\right) d t_{1},
$$

where

$$
\begin{aligned}
\mathbf{S}_{1}\left(\mathbf{U}_{L}\left(x, t_{1}\right)\right) & =\left(\begin{array}{c}
-\gamma \alpha_{1 L}\left(x, t_{1}\right) \beta_{L}^{2}\left(x, t_{1}\right) \\
\gamma \alpha_{1 L}\left(x, t_{1}\right) \beta_{L}^{2}\left(x, t_{1}\right) \\
0
\end{array}\right), \\
\mathbf{U}_{L}(x, t) & =e^{t \hat{M}_{1}}\left(\begin{array}{lll}
1 & h g(x) & 1
\end{array}\right)^{T} .
\end{aligned}
$$

By substituting (10) into (26) and using the expansion for $e^{t \hat{M}_{1}}$ as in [3], we have

$$
\mathbf{U}_{L}(x, t)=\left(\begin{array}{c}
1 \\
\beta_{L}(x, t) \\
1
\end{array}\right),
$$




$$
\begin{aligned}
\beta_{L}(x, t) & =\frac{h e^{-t \gamma k}}{2 \sqrt{\pi \gamma t}} \int_{-1}^{1} g\left(x_{1}\right) \exp \left[-\frac{\left(x-x_{1}\right)^{2}}{4 \gamma t}\right] d x_{1}, \\
g\left(x_{1}\right) & =e^{1 /\left(x_{1}^{2}-1\right)} .
\end{aligned}
$$

By substituting (27) and (28) into (24-26) and after a set of calculations, we obtain

$$
\begin{aligned}
\mathbf{U}_{1}(x, t)= & \mathbf{U}_{L}(x, t)+\int_{0}^{t}\left(\begin{array}{c}
\lambda_{+}\left(t, t_{1}\right) \\
\rho\left(t, t_{1}\right) \\
\lambda_{-}\left(t, t_{1}\right)
\end{array}\right) f_{1}\left(x, t, t_{1}\right) d t_{1} \\
f_{1}\left(x, t, t_{1}\right)= & h^{2} \int_{-1}^{1} \int_{-1}^{1} \frac{g\left(x_{1}\right) g\left(x_{2}\right)}{4 \pi \sqrt{t_{1}\left(2 t-t_{1}\right)}} \exp \left\{-\frac{1}{8 \gamma}\left[\frac{\left(x_{1}-x_{2}\right)^{2}}{t_{1}}\right.\right. \\
& \left.\left.\quad+\frac{\left(-2 x+x_{1}+x_{2}\right)^{2}}{2 t-t_{1}}+16 k \gamma^{2} t_{1}\right]\right\} d x_{1} d x_{2}, \\
\lambda_{ \pm}\left(t, t_{1}\right)= & \frac{-1}{2}\left[1 \pm e^{-2 \gamma^{2}\left(t-t_{1}\right)}\right] \\
\rho\left(t, t_{1}\right)= & e^{-k \gamma\left(t-t_{1}\right)} .
\end{aligned}
$$

Most of the results found here have been obtained using Mathematica. Note that the contribution from the nonlinear coupling between the reactant and the autocatalyst given by the second term in the right-hand side of (30) tends to zero uniformly as $t \rightarrow \infty$. Therefore the Picard iteration scheme converges uniformly. Predominantly,

$$
\left\|\mathbf{U}_{1}-\mathbf{U}_{L}\right\|=\mathcal{O}\left(t^{-1}\right), \quad\left\|\mathbf{U}_{2}-\mathbf{U}_{1}\right\|=\mathcal{O}\left(t^{-2}\right), \quad \ldots .
$$

Thus the above results are valid for $0 \leq t \leq T$, where $T$ is taken appropriately so that the maximum error between the second and first approximation is of order $10^{-3}$. This will be discussed in Section 4 .

First, the solutions for $\alpha_{1}, \beta$ and $\alpha_{2}$ given by (30-33) are displayed against $x$ for different values of $t, k, \gamma$ and $h$. In each one of the following figures, the results for $\alpha_{1}, \beta$ and $\alpha_{2}$ are plotted in parts (a), (b) and (c) respectively. 
- Figure 1 displays solutions for $k=0.05, \gamma=0.2$ and $h=5$. The dot-dashed, dashed and solid curves correspond to the values of $t=10,50$ and 100 respectively. From these figures, we find that the concentration $\beta$ is sufficiently small almost everywhere for $t \geq 100$. On the other hand the concentration $\alpha_{1}$ spreads more quickly in comparison with $\alpha_{2}$. This reflects the broadening effect of the nonlinear term in the equation for $\alpha_{1}$. Thus the coupling between the autocatalyst and the reactant in the first region has the effect of broadening the domain of reaction. Nevertheless, $\alpha_{2}$ decreases more rapidly than does $\alpha_{1}$.

- Figure 2 displays solutions for $t=10, \gamma=0.2$ and $h=5$. The dash-dotted, dashed and solid curves correspond to $k=0.05$, 1 and 1.5 respectively. See that by increasing the decay rate $k$, the concentrations $\alpha_{1}$ and $\alpha_{2}$ are slightly changed. This means that for high values of the decay rate $k$ (namely for $k>1$ ), the reaction through coupling between the reactant and the autocatalyst or the interchange between the two regions is weak.

- Figure 3 displays solutions for $h=5, t=10$ and $k=0.05$. The dash-dotted, dashed and solid curves correspond to the values $\gamma=0.08,0.2$ and 0.7 respectively. We find that $\alpha_{1}$ decreases significantly for sufficiently small values of $\gamma$ in contrast to $\alpha_{2}$ whereas $\beta$ increases almost everywhere as $\gamma$ decreases. This reflects the dominance of the coupling between the autocatalyst and the reactant in the first cell when the rate of interchange of the reactant across the walls is sufficiently small.

- Figure 4 displays solutions for $t=10, k=0.05$ and $\gamma=0.2$. The dash-dotted, dashed and solid curves correspond to $h=3$, 5 and 6 respectively. These show that increasing the initial 


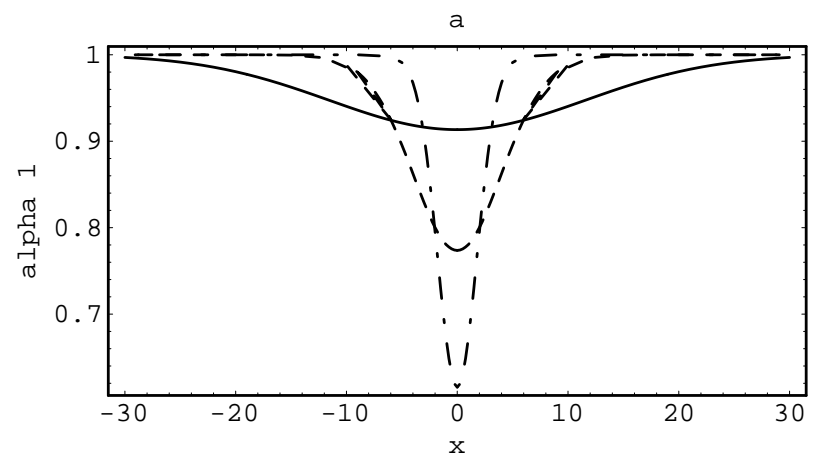

(a)

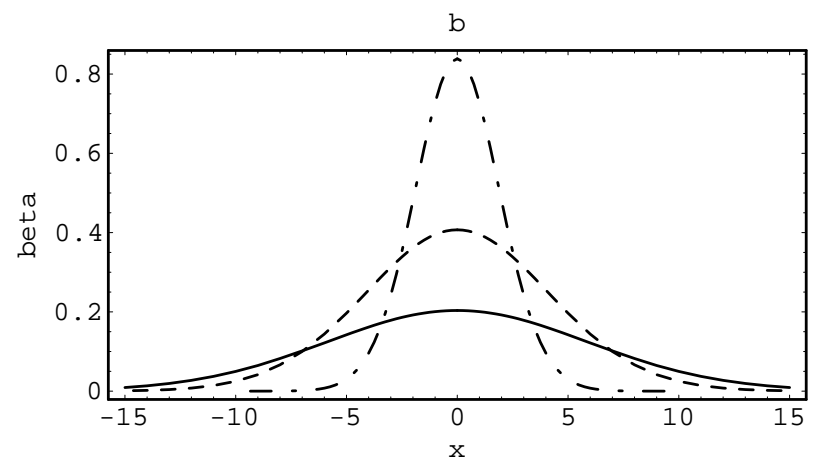

(b)

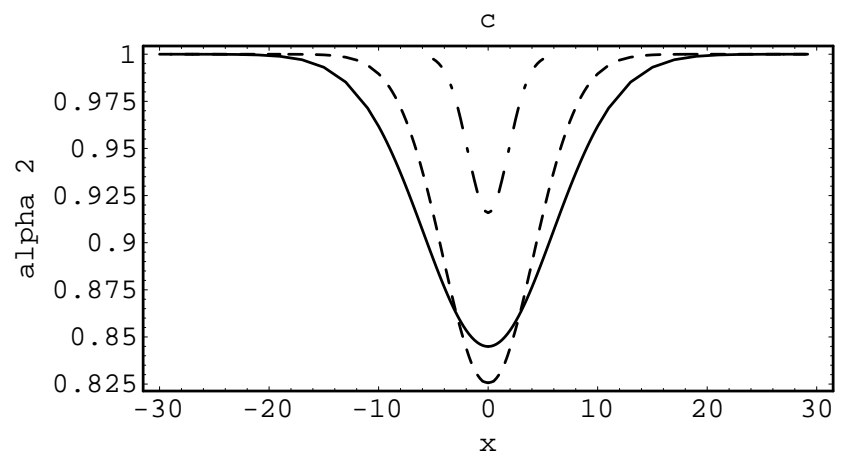

(c)

Figure 1: The solutions for $\alpha_{1}, \beta$ and $\alpha_{2}$ are displayed against $x$ in (a), (b) and (c) respectively for $h=5, k=0.05$ and $\gamma=0.2$ : $(-\cdot) t=10 ;(--) t=50 ;(-) t=100$. 


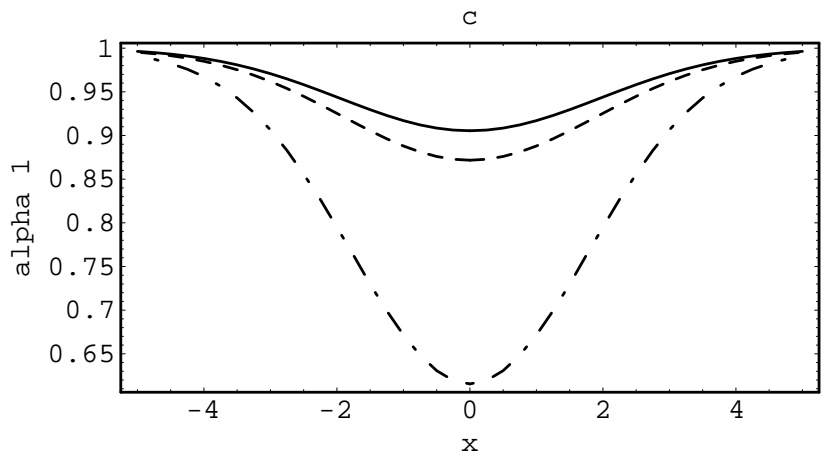

(a)

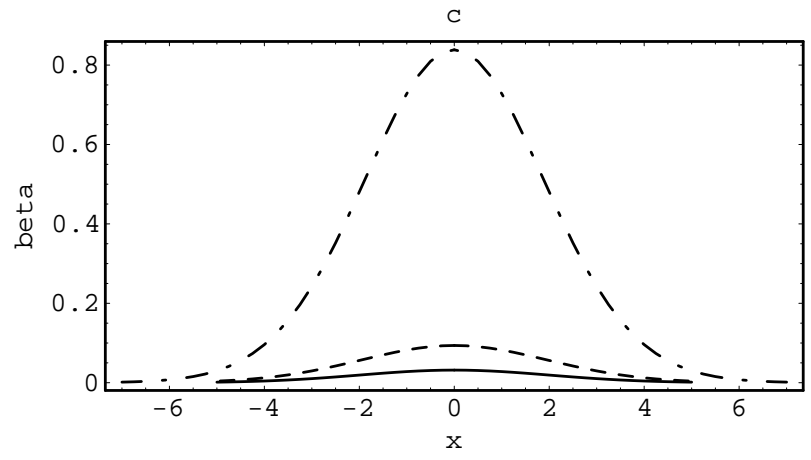

(b)

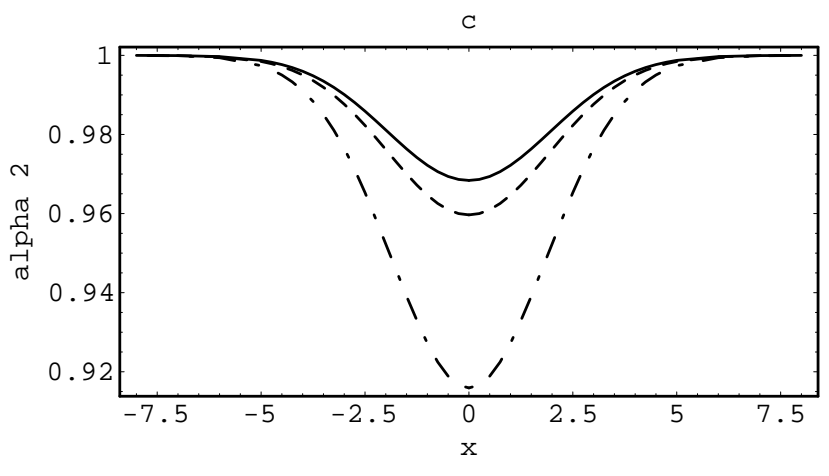

(c)

Figure 2: The solutions for $\alpha_{1}, \beta$ and $\alpha_{2}$ are displayed against $x$ in (a), (b) and (c) respectively for $h=5, t=10$ and $\gamma=0.2$ : (---) $k=0.05 ;(-) k=1 ;(-) k=1.5$. 


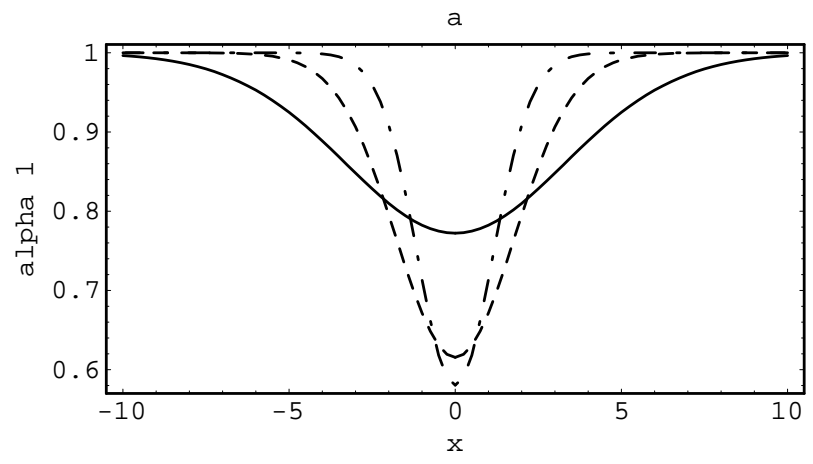

(a)

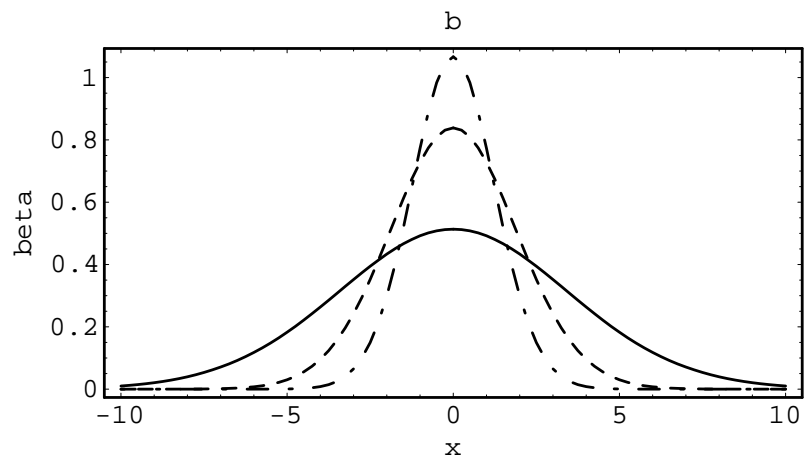

(b)

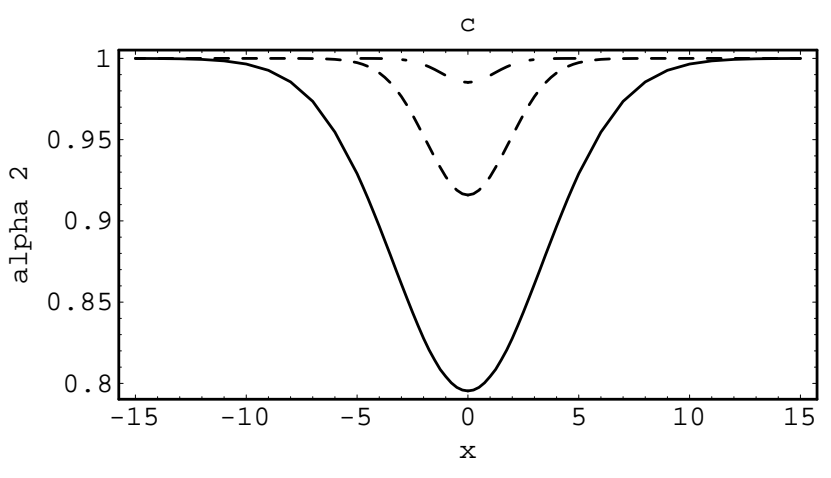

(c)

Figure 3: The solutions for $\alpha_{1}, \beta$ and $\alpha_{2}$ are displayed against $x$ in (a), (b) and (c) respectively for $h=5, t=10$ and $k=0.05$ : (-.-) $\gamma=0.08 ;(-) \gamma=0.2 ;(-) \gamma=0.7$. 
concentration of the autocatalyst activates the reaction.

In Figures 5-8, the solutions (30-33) are displayed against time $t$ for different values of $x, k, \gamma$ and $h$.

- Figure 5 displays solutions for $h=5, k=0.05$ and $\gamma=0.5$. The dash-dotted, dashed and solid curves correspond to $x=3$, 4 and 5 respectively. See that $\alpha_{1}, \beta$ and $\alpha_{2}$ oscillate with time. The oscillation is meaningful for small values of $x$ (the core of the reaction vessel). This phenomena is strong in the autocatalyst concentration.

- Figure 6 displays solutions for $h=5, x=5$ and $\gamma=0.5$. The dash-dotted, dashed and solid curves correspond to $k=0.5$, 1 and 1.5 respectively. Observe that the rapid decay of the autocatalyst has a passive effect on the oscillation phenomena. For $k \gg 1$, one finds the concentrations decay mainly linearly.

- Figure 7 displays solutions for $h=5, k=0.05$ and $x=$ 5. The dash-dotted, dashed and solid curves correspond to $\gamma=0.4,0.5$ and 0.7 respectively. See that the oscillations are significant in increasing the rate of interchange of the reactant between the two regions.

- Figure 8 displays solutions for $\gamma=0.5, k=0.05$ and $x=5$. The dash-dotted, dashed and solid curves correspond to $h=4$, 5 and 6 respectively. The variation of the initial concentration of the autocatalyst has a slight effect on the observed oscillation.

Secondly, consider the case $\gamma>1$ where the results obtained as given by (30-33) hold but $\left(t, t_{1}\right) \rightarrow \gamma^{-1}\left(t, t_{1}\right)$. We visualize the 


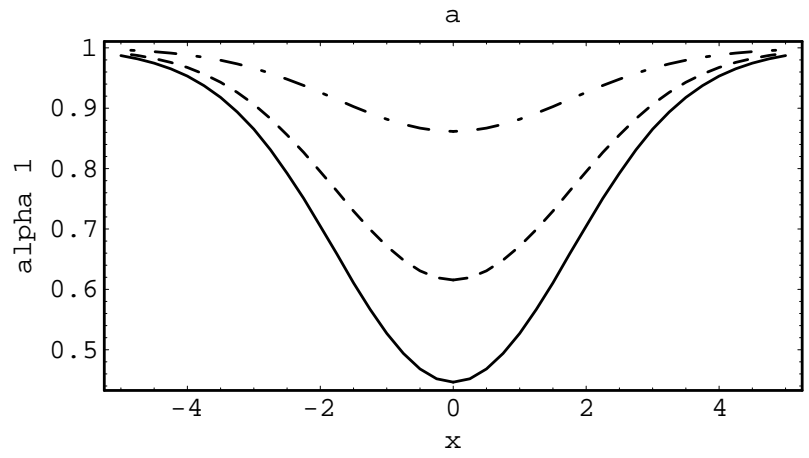

(a)

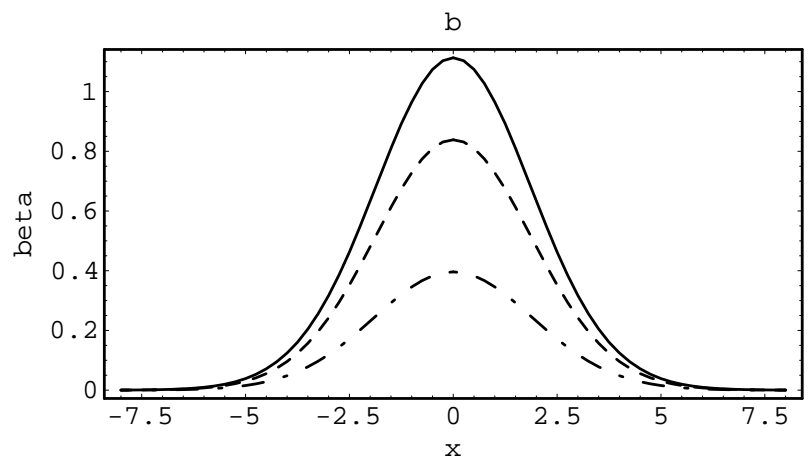

(b)

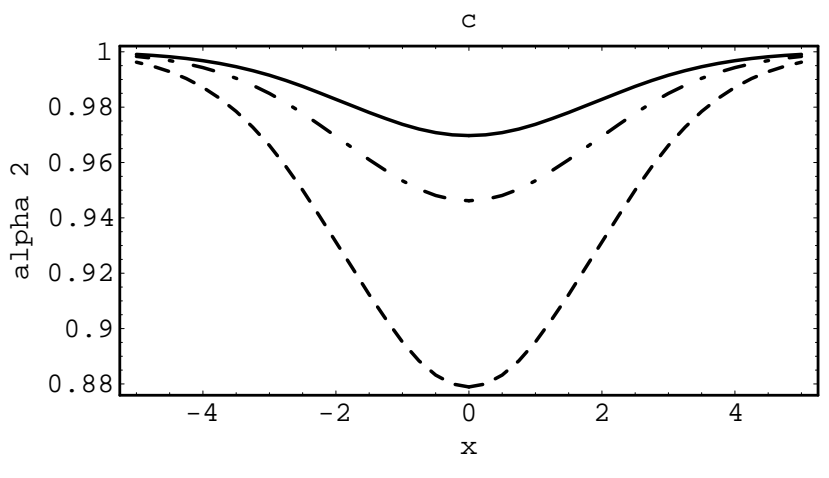

(c)

Figure 4: The solutions for $\alpha_{1}, \beta$ and $\alpha_{2}$ are displayed against $x$ in (a), (b) and (c) respectively for $t=1, k=0.05$ and $\gamma=0.2:(-\cdot-)$ $h=3 ;(-) h=5 ;(-) h=6$. 


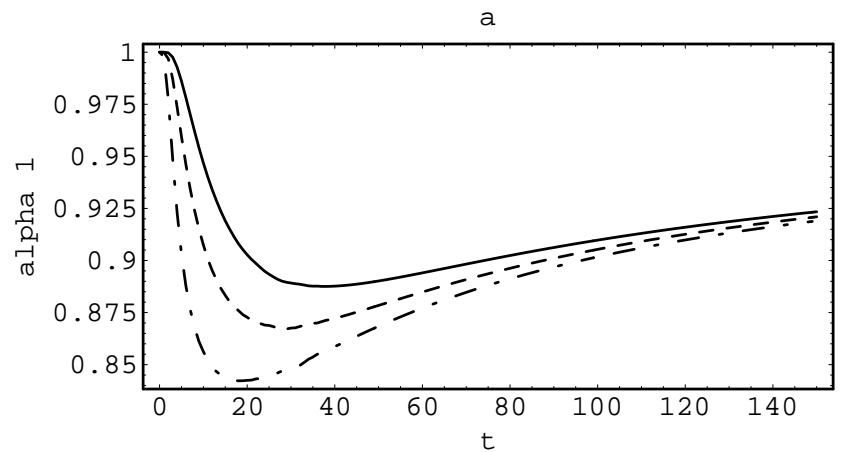

(a)

b

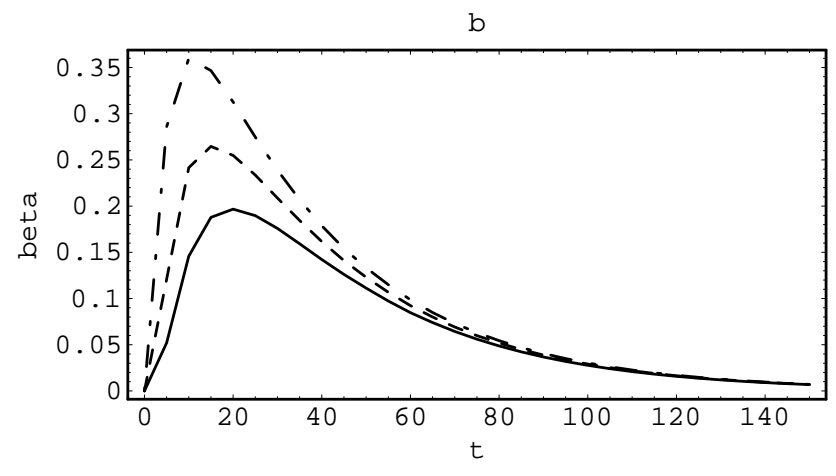

(b)

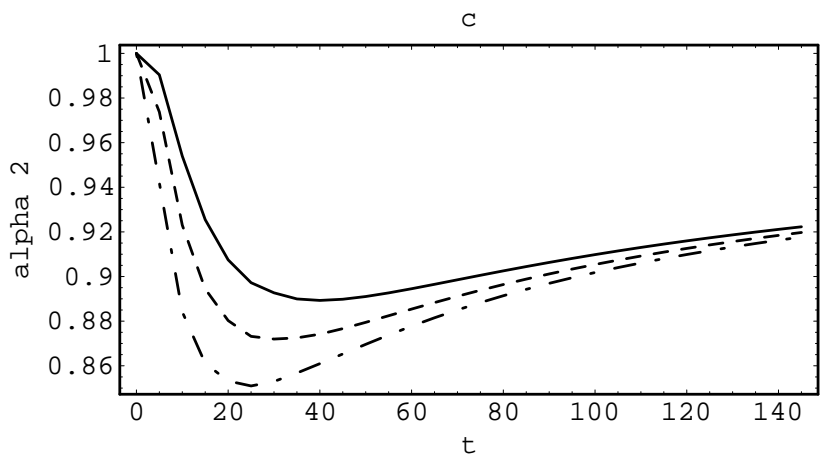

(c)

Figure 5: The solutions for $\alpha_{1}, \beta$ and $\alpha_{2}$ are displayed against $t$ in (a), (b) and (c) respectively for $h=5, k=0.05$ and $\gamma=0.5:(-\cdot-)$ $x=3 ;(-) x=4 ;(-) x=5$. 
3 The first approximation to the solution of the problem for $\gamma<1 \mathrm{E} 18$

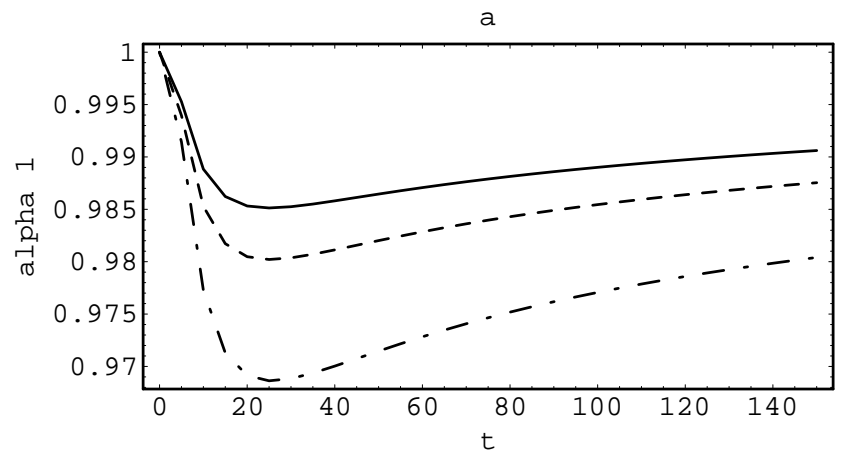

(a)

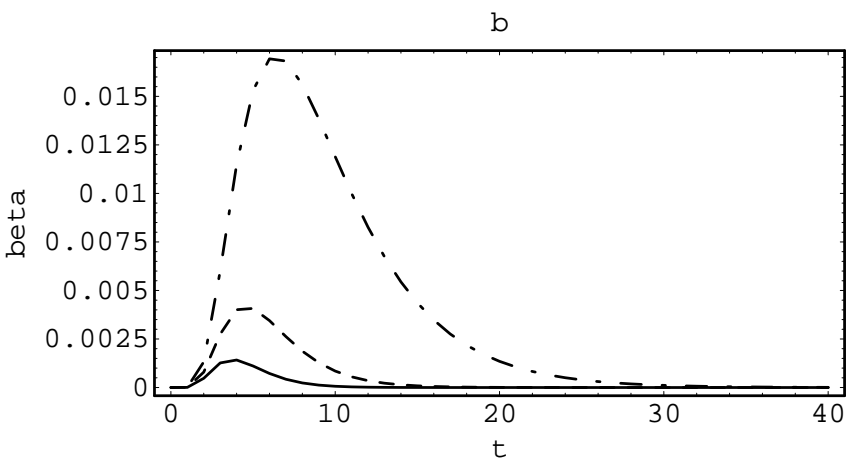

(b)

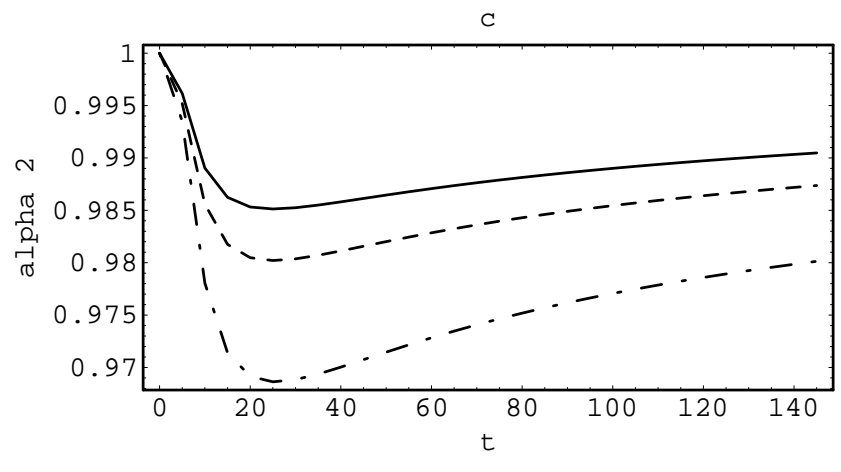

(c)

Figure 6: The solutions for $\alpha_{1}, \beta$ and $\alpha_{2}$ are displayed against $t$ in (a), (b) and (c) respectively for $h=5, x=5$ and $\gamma=0.5$ : (-.-) $k=0.5 ;(-) k=1 ;(-) k=1.5$. 
(a)

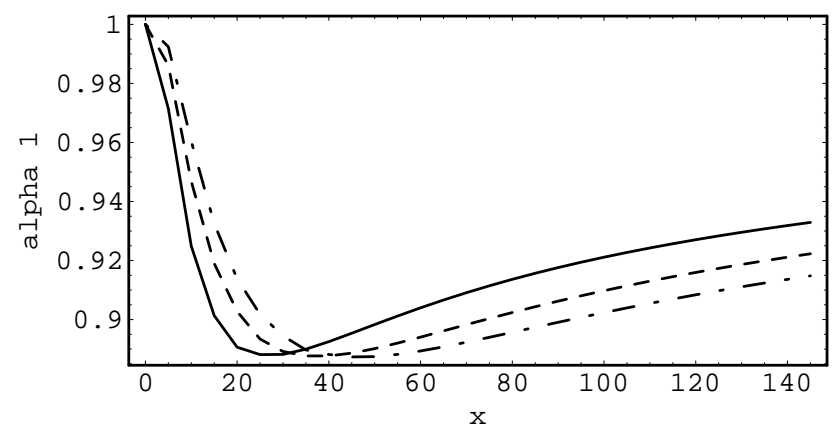

b

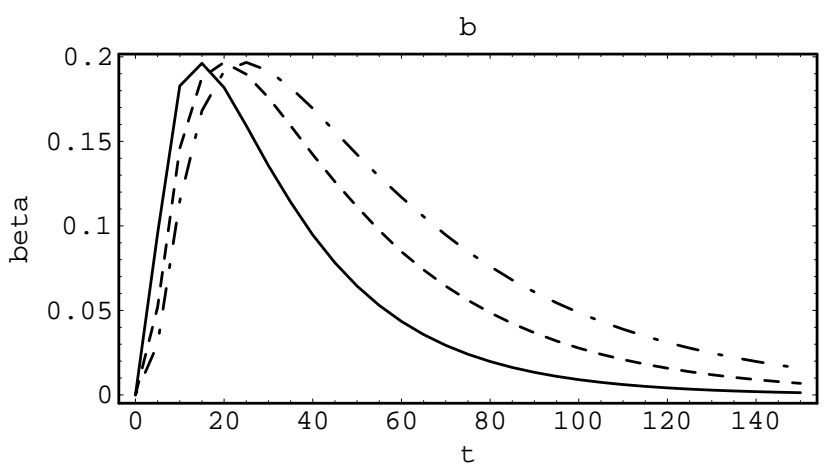

(b)

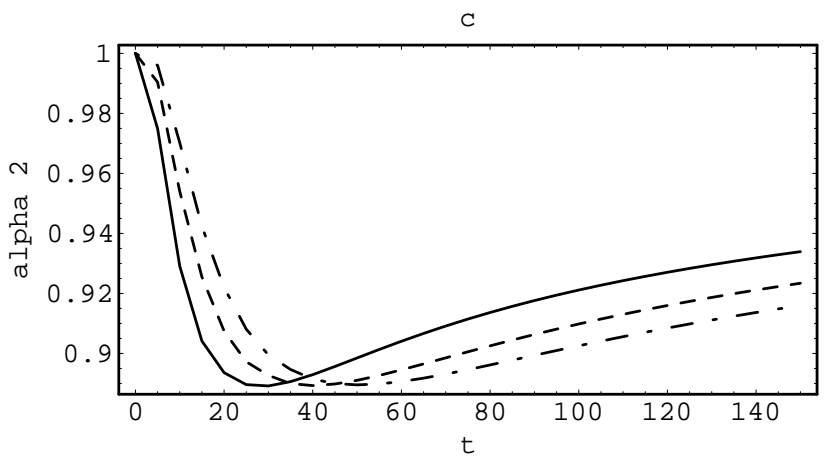

(c)

Figure 7: The solutions for $\alpha_{1}, \beta$ and $\alpha_{2}$ are displayed against $t$ in (a), (b) and (c) respectively for $h=5, k=0.05$ and $x=5$ : (---) $\gamma=0.4 ;(--) \gamma=0.5(-) \gamma=0.7$. 
3 The first approximation to the solution of the problem for $\gamma<1 \mathrm{E} 20$

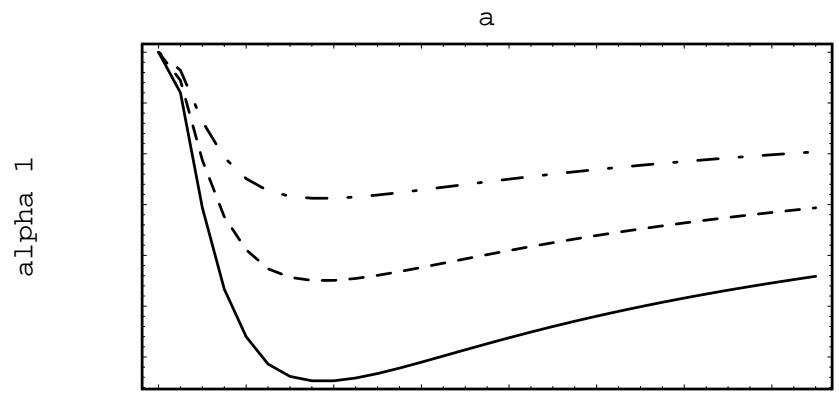

(a)

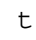

b

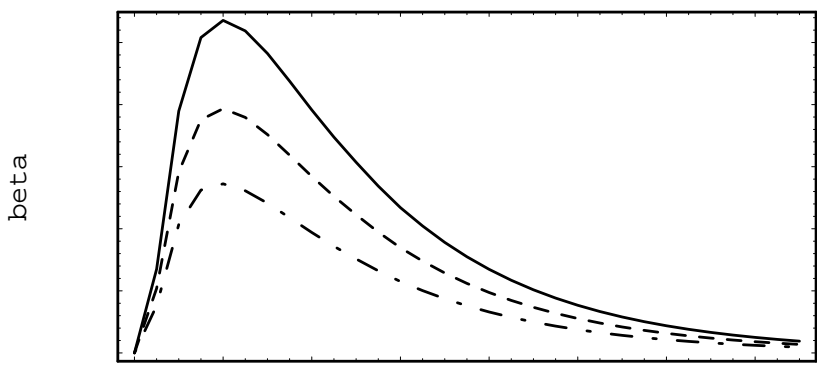

(b)

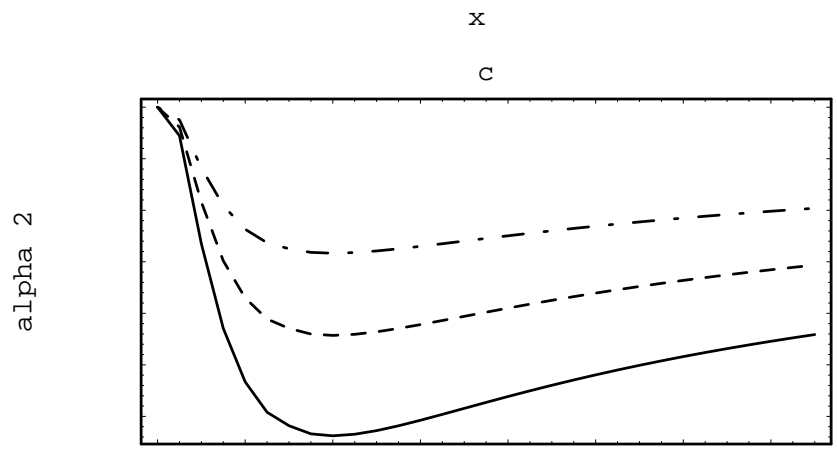

(c)

t

Figure 8: The solutions for $\alpha_{1}, \beta$ and $\alpha_{2}$ are displayed against $t$ in (a), (b) and (c) respectively for $\gamma=0.5, k=0.05$ and $x=5:(-\cdot-)$ $h=4 ;(-) h=5 ;(-) h=6$. 
effects of the high rate of interchange of the reactant $A$ on the reaction, in the following figures. Figure 9 displays the results (30$33)$, with $\left(t, t_{1}\right) \rightarrow \gamma^{-1}\left(t, t_{1}\right)$ against $x$ for $k=0.05, h=5$ and $\gamma=2$, The dash-dotted, dashed and solid curves correspond to $t=10$, 50 and 100 respectively. See that the replenishment of the reactant is mainly the same in the two regions. This is in contrast to the case of small values of $\gamma$. In Figure 10 the results are plotted against $t$ for $k=0.05, h=5$ and $\gamma=5$. The dash-dotted, dashed and solid curves correspond to $x=0.5,1$ and 5 respectively. The oscillation of the concentrations is weakened for $\gamma>1$.

\section{The second approximation and maximum error estimate}

Notice that the problem under consideration admits some conservation laws for some special values of the parameters $k$ and $\gamma$. For $k=0$, the first conservation law is that the sum of the concentrations satisfies the linear diffusion equation

$$
\left(\partial_{t}-\partial_{x}^{2}\right) \Phi_{1}=0, \quad \Phi_{1}=\alpha_{1}+\beta_{1}+\alpha_{2} .
$$

For $k=2 \gamma$, we have the second conservation law

$$
\left(\partial_{t}-\partial_{x}^{2}\right) \Phi_{2}=0, \quad \Phi_{2}=\left(\alpha_{1}+\beta_{1}-\alpha_{2}\right) e^{-2 \gamma t} .
$$

We now evaluate $\Phi_{1}$ or $\Phi_{2}$ exactly from (35) and (36) by using the initial conditions under consideration and as given approximately in Section 3. We find that the error between the exact and approximate values for these functions vanishes identically. Thus we are led to calculate the second approximation and give a maximum error estimate between the first and second approximations. The second 


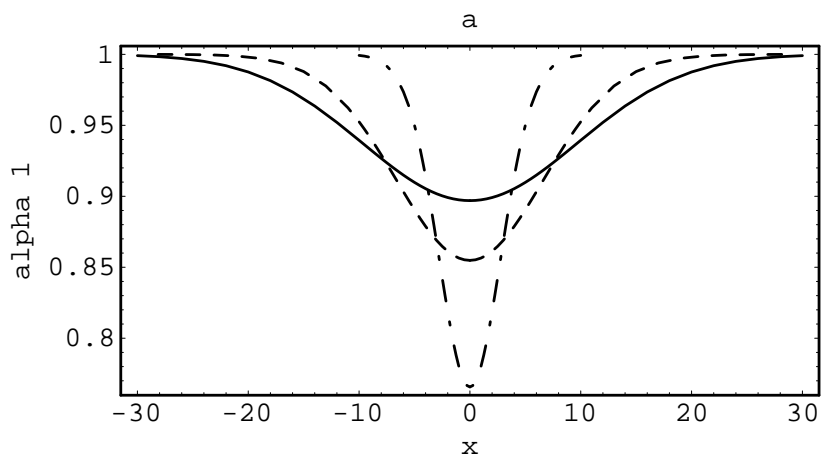

(a)

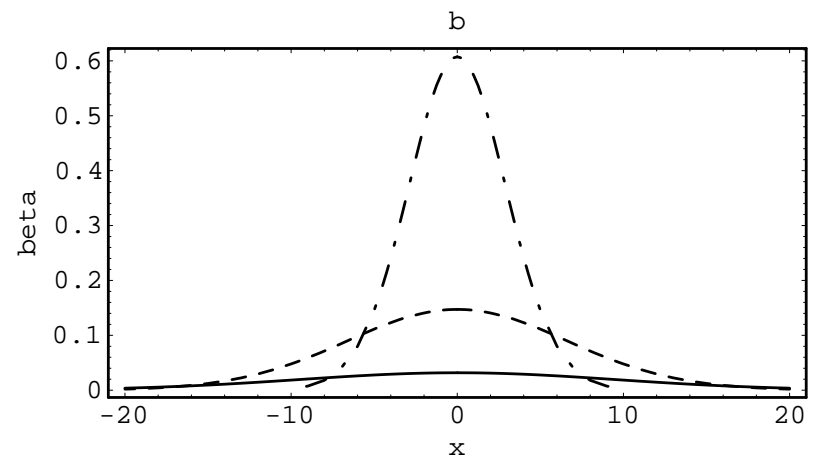

(b)

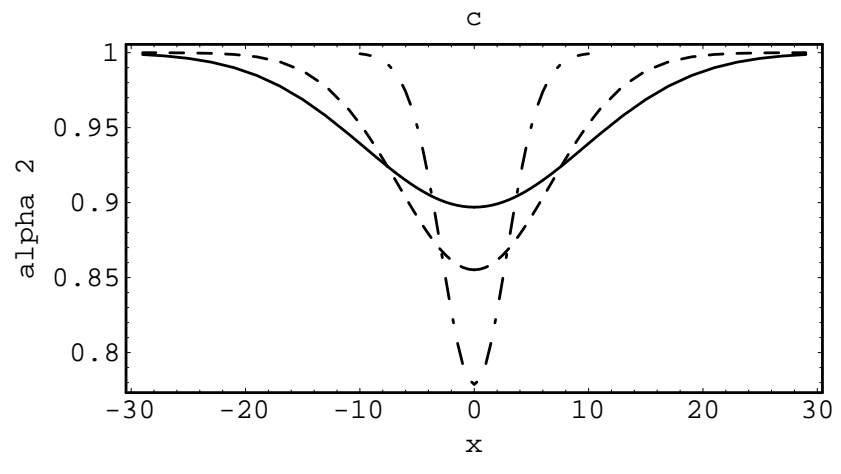

(c)

Figure 9: The solutions for $\alpha_{1}, \beta$ and $\alpha_{2}$ are displayed against $x$ in (a), (b) and (c) respectively for $h=5, k=0.05$ and $\gamma=0.2$ : $(--) t=10 ;(--) t=50 ;(-) t=100$. 
a

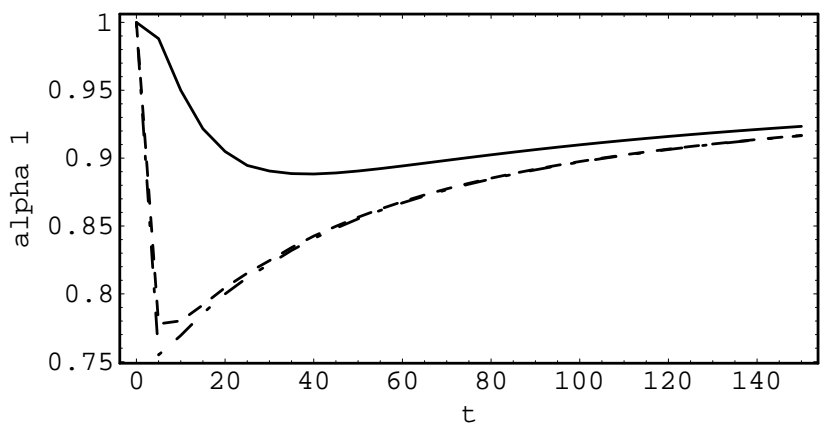

(a)

b

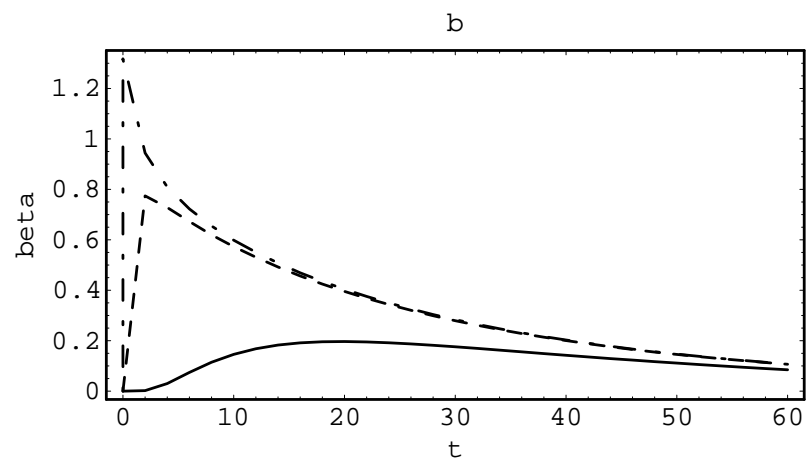

(b)

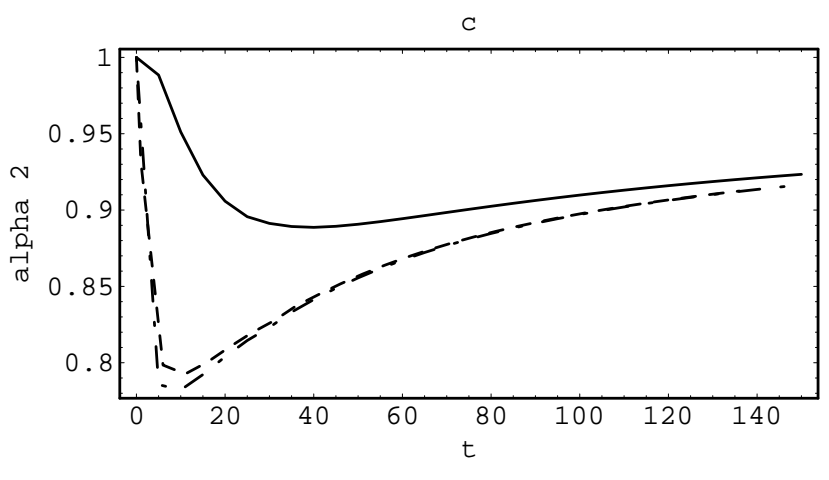

(c)

Figure 10: The solutions for $\alpha_{1}, \beta$ and $\alpha_{2}$ are displayed against $t$ in (a), (b) and (c) respectively for $h=5, k=0.05$ and $\gamma=5$ : (---) $x=0.5 ;(-) x=1 ;(-) x=5$. 
approximation is

$$
\begin{gathered}
\mathbf{U}_{2}(x, t)=\mathbf{U}_{L}(x, t)+\int_{0}^{t} e^{\left(t-t_{1}\right) \hat{M}_{1}} \mathbf{S}_{1}\left(\mathbf{U}_{1}\left(x, t_{1}\right)\right) d t_{1}, \\
\mathbf{S}_{1}\left(\mathbf{U}_{1}\left(x, t_{1}\right)\right)=\left(\begin{array}{c}
-\gamma \alpha_{1}^{(1)}\left(x, t_{1}\right)\left(\beta^{(1)}\right)^{2}\left(x, t_{1}\right) \\
\gamma \alpha_{1}^{(1)}\left(x, t_{1}\right)\left(\beta^{(1)}\right)^{2}\left(x, t_{1}\right) \\
0
\end{array}\right),
\end{gathered}
$$

where $\alpha_{1}^{(1)}, \alpha_{2}^{(1)}$ and $\beta^{(1)}$ are the components of $\mathbf{U}_{1}$ given by (30-33). By substituting these results into (37) and (38), we get for $\gamma<1$ with $t \rightarrow \gamma t$

$$
\begin{aligned}
& \mathbf{U}_{2}(x, t) \\
& =\mathbf{U}_{L}(x, t)+\int_{0}^{t}\left(\begin{array}{c}
\lambda_{+}\left(t, t_{1}\right) \\
\rho\left(t, t_{1}\right) \\
\lambda_{-}\left(t, t_{1}\right)
\end{array}\right) f_{1}\left(x, t, t_{1}\right) d t_{1} \\
& +2 \int_{0}^{t} \int_{0}^{t_{1}}\left(\begin{array}{c}
\lambda_{+}\left(t, t_{1}\right) \rho\left(t, t_{1}\right) \\
\rho\left(t, t_{1}\right) \rho\left(t_{1}, t_{2}\right) \\
\left.\lambda_{-}\left(t, t_{1}\right) \rho\left(t_{1}, t_{2}\right)\right)
\end{array}\right) f_{2}\left(x, t, t_{1}, t_{2}\right) d t_{2} d t_{1} \\
& +\int_{0}^{t} \int_{0}^{t_{1}}\left(\begin{array}{c}
\lambda_{+}\left(t, t_{1}\right) \lambda_{+}\left(t_{1}, t_{2}\right) \\
\rho\left(t, t_{1}\right) \lambda_{+}\left(t_{1}, t_{2}\right) \\
\lambda_{-}\left(t, t_{1}\right) \lambda_{+}\left(t_{1}, t_{2}\right)
\end{array}\right) f_{3}\left(x, t, t_{1}, t_{2}\right) d t_{2} d t_{1} \\
& +f_{2}\left(x, t, t_{1}, t_{2}\right), \begin{array}{c}
1 \\
= \\
h^{3}
\end{array} \int_{-1}^{1} \int_{-1}^{1} \int_{-1}^{1} \frac{\sqrt{\gamma} \prod_{i=1}^{3} g\left(x_{i}\right) d x_{i}}{8 \sqrt{\pi^{3} t_{2}\left(t t^{*}-2 t_{1}^{2}\right)} \times} \\
& \quad \times \exp \left(\frac { - 1 } { 8 \gamma t _ { 2 } ( t t ^ { * } - 2 t _ { 1 } ^ { 2 } ) } \left\{( t t ^ { * } - 2 t _ { 1 } ^ { 2 } ) \left[\left(x_{1}-x_{2}\right)^{2}\right.\right.\right. \\
& \left.\quad+8 k \gamma^{2} t_{2}\left(t_{1}+2 t_{2}\right)\right]+2 t_{2}\left[\left(x_{3}-x\right)^{2}\left(2 t_{1}-t_{2}\right)\right. \\
& \left.\left.\left.\quad+\left(x^{*}-2 x\right)^{2}\left(t-t_{1}\right)\right]\right\}\right),
\end{aligned}
$$




$$
\begin{aligned}
& f_{3}\left(x, t, t_{1}, t_{2}\right) \\
= & h^{4} \int_{-1}^{1} \int_{-1}^{1} \int_{-1}^{1} \int_{-1}^{1} \frac{\sqrt{2} \prod_{i=1}^{4} g\left(x_{i}\right) d x_{i}}{16 \pi^{2} \sqrt{t_{1} t_{2}} \sqrt{2 t\left(3 t_{1}-t_{2}\right)-t_{1} t^{*}}} \times \\
\times & \exp \left(\frac{-\left(x_{1}-x_{2}\right)^{2}}{8 \gamma t_{2}}+\frac{-1}{q}\left\{2\left(t t^{*}-2 t_{1}^{2}\right)\left(x_{3}^{2}+x_{4}^{2}\right)\right.\right. \\
& +t_{1}\left[4 x^{2}\left(3 t_{1}-t_{2}\right)-4 x x^{*} t_{1}+\left(x^{*}\right)^{2}\left(2 t-t_{1}\right)\right] \\
& -4 x_{4} t_{1}\left[x\left(2 t_{1}-t_{2}\right)+x^{*}\left(t-t_{1}\right)\right]-4 x_{3}\left[x_{4}\left(2 t_{1}-t_{2}\right) \times\right. \\
& \left.\left.\left.\times\left(t-t_{1}\right)+t_{1}\left(x\left(2 t_{1}-t_{2}\right)+x^{*}\left(t-t_{1}\right)\right)\right]\right\}\right) \\
q= & 8 \gamma t_{1}\left(2 t\left(3 t_{1}-t_{2}\right)-t_{1} t^{*}\right)-2 k \gamma\left(t_{1}+t 2\right)
\end{aligned}
$$

where $x^{*}=x_{2}+x_{3}$ and $t^{*}=4 t_{1}-t_{2}$. For $\gamma \geq 1$ the above results hold but $\left(t_{1}, t_{2}\right) \rightarrow \gamma^{-2}\left(t_{1}, t_{2}\right)$. After the results (30-33) which are shown in Figures 1-10, one finds that the maximum or the minimum of the concentrations occurs at $x=0$. Therefore we confine ourselves to evaluating the error estimate between the first and second approximation at $x=0$, namely $R_{i}(t)=\left|u_{i}^{(2)}(0, t)-u_{i}^{(1)}(0, t)\right|$, where $u_{i}$ is $\alpha_{1}, \beta$ or $\alpha_{2}$. The results (30-33) and (39-41) are used to evaluate the function $R_{i}(t)$ and the results are shown in Figure 11 for $h=2, k=0.5$ and $\gamma=0.4$. See that the maximum error oscillates, mainly increasing with $t$ up to $t \approx 10$ (dimensionless) and then decreases to some value less than $2 \times 10^{-3}$ where it saturates. The error depends significantly on the value of $h$ (or the initial concentration of the autocatalyst): it increases with $h$ increasing. The error remains of the same order for the values of $h$ considered here up to $h=5$. However, the error estimate depends weakly on the values of $k$ and $\gamma$.

In Figures 1-4 observe that the minimum (or maximum) value of $\alpha_{i}$ (or $\beta$ ) does not vary with time. This means that no initiation to travelling waves occurs. In fact, when analysing the set of equations (6-9), we find that the diffusion term assumes an unbounded 


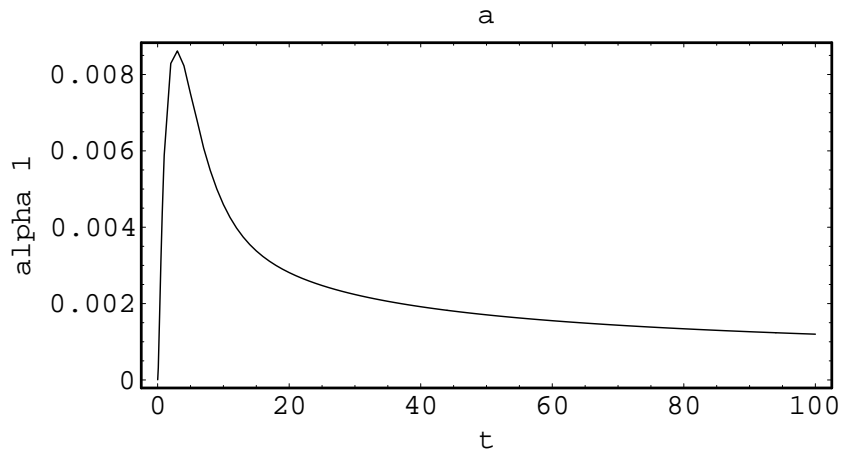

(a)

b

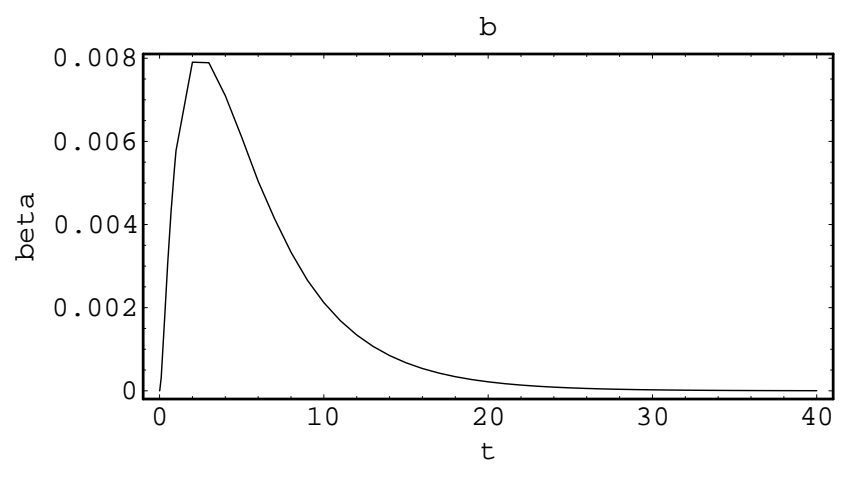

(b)

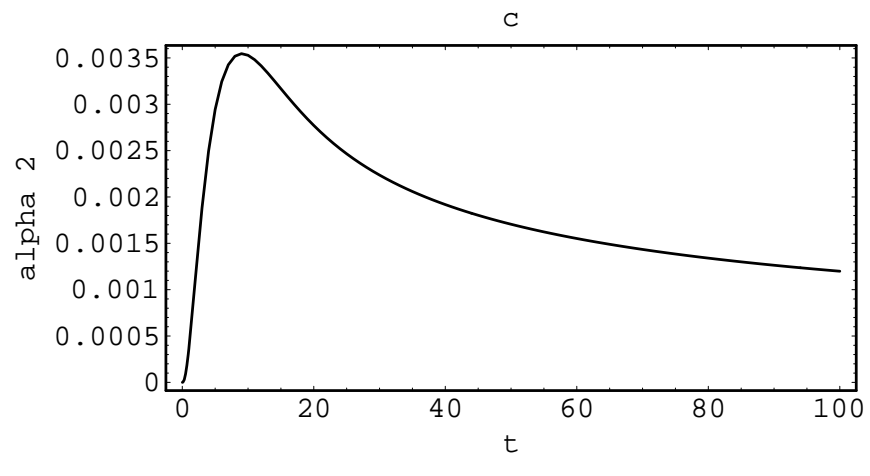

(c)

FiguRE 11: The maximum error estimate between the second and the first approximation for $\alpha_{1}, \beta$ and $\alpha_{2}$ is displayed against $t$ in (a), (b) and (c) respectively for $h=2, k=0.5$ and $\gamma=0.4$. 
propagation speed. The effect of the presence of the linear terms in $\alpha_{i}$ (or $\beta$ ) is to accelerate or decelerate the reaction, while the nonlinear coupling terms $\alpha_{i} \beta^{2}$ play the role of enlarging the domain of reaction. This is shown in Figures 1-4. For example in Figure 1, see that the concentration $\alpha_{1}$ is equal to its initial value (namely 1 ) on $|x|>30$. That is, the reaction holds for $|x| \leq 30$. But the concentration $\alpha_{2}$ differs from 1 on $|x| \leq 20$. Thus the presence of the nonlinear coupling term results in an enlarging of the reaction domain. As no initiation to travelling waves occurs, there exists no realistic permanent travelling wave solution for (6-9). We think that a realistic permanent travelling wave is an intrinsic property of the nonlinear evolution equations. This will be discussed in the next section.

\section{On the existence of realistic permanent travelling wave solutions}

First, consider a single nonlinear evolution equation of the form

$$
u_{t}=F\left(x, t, u, u_{x}, u_{x x}, \ldots\right)
$$

where $F$ is analytic in its arguments. When the function $F$ is linear in $u_{x}$, namely

$$
F=-\nu u_{x}+F^{*}\left(x, t, u, u_{x x}, \ldots\right),
$$

then in the solution of (43), a travelling wave initiates with a speed which tends to be ultimately $\nu$. Thus a realistic permanent travelling wave solution (RPTWS) for (43) exists. The wave travels at speed $\nu$. Now we exclude this case and suggest the following proposition. 
Proposition 6 A sufficient condition for an evolution equation of the form (43) to admit a RPTWS is the existence of a PTWS $U(z) \not \equiv 0$ for (43) such that its solution is invariant under the auto-Bäcklund transformation (ABT)

$$
u(x, t)=U(Z)+\bar{u}(x, t), \quad \bar{u} \not \equiv 0, \quad Z=x-c t,
$$

where $c$ is the speed of the travelling wave. But if the ABT exists only with $U(z)=0$, then no RPTWs exists. It is understood that in the $\mathrm{ABT}$ both $u$ and $\bar{u}$ satisfy (43).

To clarify this proposition, we give some examples. If the function $F$ in the right-hand side of (43) is

$$
F=u_{x x}+\lambda u(1-u)
$$

then (43) and (46) become the Fisher equation. In this case the solution of these equations is invariant under (45) only when

$$
U(Z) \bar{u}(x, t)=0 .
$$

Thus (45) holds when $U(z)=0$ and no RPTws for (43) and (46) exists. We mention that the solution $\bar{u}=0$ for (45) yields the hypothetical PTws $u=U(Z)$. If the function

$$
F=u_{x x}+u u_{x}
$$

then (43) and (48) yields Burgers' equation. The condition of invariance of the solution of (43) and (48) under (45) is

$$
\frac{d U}{d z} \bar{u}+U(z) \bar{u}_{x}=0 .
$$

We find that (49) solves for $U(z)=U_{0} e^{\lambda z}$ and $\bar{u}=g(t) e^{-\lambda x}$, where $U_{0}$ is an arbitrary constant and $g(t)$ is an arbitrary function. Thus 
Burgers' equation admits a RPTws. Thus a sufficient condition that the evolution equation (43) admits a RPTWs is that $F$ is an explicit function in $u_{x}$ (the advection term). The necessary condition for the existence of a RPTWS may arise from the initial or boundary conditions. We note from the problem studied in Section 5 that the set of equations (6-8) do not verify the sufficient condition for a RPTWS to exist. On the other hand, the initial conditions (9) on the infinite domain are not periodic. Thus no RPTws exists. This statement holds for dynamical systems of infinite size which do not satisfy the sufficient condition and with non-periodic initial conditions.

For dynamical systems of finite size which do not satisfy the sufficient condition, the situation is different. Self-oscillations are induced by periodic boundary conditions [21]. For soft (Dirichlet) boundary conditions, the modes generated are mainly damping. The most dominant mode(s) initiate(s) travelling waves. In this case a RPTwS exists. For rigid (Neumann) boundary conditions, some modes generated are growing and symmetry breaking occurs leading probably to chaotic behaviour [4].

\section{Conclusions}

Solutions of the initial value problem for the two region cubic autocatalytic reaction model have been obtained. These solutions have been found by truncating the Picard iterative sequence of solutions constructed after the exact ones. We have shown that the concentrations of the reactants spread towards the walls of the reaction vessel as time increases. The spreading of either the reactant or the autocatalyst depends significantly on $\gamma$ but weakly on the parameter values of $h$ and $k$. It has been also shown that the concentrations oscillate with time in the core of the reaction vessel for a small 
time period. This phenomenon is shown to be more significant in the autocatalyst than in the reactant and is moderately affected by varying the control parameters $\gamma, k$ and $h$. We have found that no initiation to travelling waves occurs whatever the concentrations of the reactant or the autocatalyst.

\section{References}

[1] H. I. Abdel-Gawad. Solution of a class of partial differential equations. some diffusion equations. Math. Meth. In the Appl. Sci., 12:221-228, 1990. E6

[2] H. I. Abdel-Gawad. Solution of the equation $\partial_{t}^{n}=\hat{l}(x, t) f(x, t)+s(x, t)$. exact solution of some partial differential equations in mechanics and physics. Math. Meth. In the Appl. Sci., 14:615-633, 1991. E6

[3] H. I. Abdel-Gawad and A. K. El-sharee. An approach to solutions of coupled semilinear partial differential equations with applications. Math. Meth. In the Appl. Sci., 23:845-864, 2000. E6, E9

[4] V. M. Eguíluz, P. AlstrØm, E. Hernández-García, and Piro. Average patterns of spatiotemporal chaos :a boundary effect. Phys.Rev., 59:2822-2826, 1999. E29

[5] R. J. Field and M. Burger. Oscillations and travelling waves in chemical systems. J.Wiley, New York, 1985. E3

[6] M. S. Garley, E. Jones, and G. Stedman. Travelling reaction fronts in the nitric acid oxidation of thiocyanate. Phil. Trans. R. Soc. Lond., A337:237-247, 1991. E3 
[7] R. J. Gowland, E. Jones, and G. Stedman. Moving reaction zone chemistry: the hydroxylamine, sodium thiocyanate, nitricacid system. react. Kinet. Catal. Lett., 32:15-19, 1986. E3

[8] J. A. Leach, J. H. Merkin, and S. K. Scott. An analysis of two cell coupled nonlinear chemical oscillator. Dynam. Stability Syst., 6:341-366, 1991. E3

[9] J. A. Leach, J. H. Merkin, and S. K. Scott. Two-cell coupled cubic autocatalator the effect of the uncatalysed reaction. Dynam. Stability Syst., 7:245-266, 1992. E3

[10] J. Maselko and K. Showalter. Chemical waves on spherical surfaces. Nature, 339:609-611, 1989. E3

[11] J. Maselko and K. Showalter. Chemical waves in inhomogeneous excitable media. Physica D, 49:21-32, 1991. E3

[12] J. H. Merkin and D. J. Needham. Propagating reaction-diffusion waves in a simple isothermal autocatalytic chemical system. J. Eng. Math, 23:343-356, 1989. E3

[13] J. H. Merkin and D. J. Needham. The development of traveling waves in a simple autocatalytic chemical system. ii cubic autocatalysis with quadratic and linear decay. Proc. $R$. Soc. Lond., A430:315-345, 1990. E3

[14] J. H. Merkin and D. J. Needham. Iv quadratic autocatalysis with quadratic decay. Proc. R. Soc. Lond., A434:531-554, 1991. E3

[15] J. H. Merkin, D. J. Needham, and S. K. Scott. The development of traveling waves in a simple isothermal 
chemical system. i. quadratic autocatalysis with linear decay. Proc. R. Soc. Lond, A 424:187-209, 1989. E3

[16] J. H. Merkin, D. J. Needham, and S. K. Scott. Coupled reaction diffusion waves in an isothermal autocatalytic chemical system. IMA J. Appl. Math, 50:43-76, 1993. E3

[17] M. J. Metcalf, J.H. Merkin, and S. K. Scott. Reaction diffusion waves in coupled isothermal autocatalytic chemical systems. IMA J. Appl. Math., 51:269-298, 1993. E3, E4, E5

[18] D. J. Needham. A note on the global asymptotic stability of the unreacting state in a simple model for quadratic autocatalysis with linear decay. Appl. Math. Phys. ZAMP, 42:455-459, 1991. E3

[19] G. Pota, I. Lengyel, and G. J. Basza. Travelling waves in the acidic nitrate-iron (ii) reaction: analytical description of the wave velocity. Phys. Chem., 95:4379-4381, 1991. E3

[20] S. K. Scott. Isolas mushrooms and oscillations in isothermal autocatalytic reaction diffusion equations. Chem. Eng. Sci., 42 No.2:307-315, 1987. E3

[21] E. Sel'kov. Self-oscillations in glycolysis. European J. Biology, 4:79-86, 1968. E29

[22] D. Winston, M. Arora, J. Maselko, V.Gaspar, and K. Showlter. Cross-membrane coupling of chemical spatiotemporal patterns. Nature, 351:132, 1991. E3 